\title{
HOMOLOGICAL DIMENSIONS IN A MORITA CONTEXT WITH APPLICATIONS TO SUBIDEALIZERS AND FIXED RINGS
}

\author{
PHILIPPE LOUSTAUNAU AND JAY SHAPIRO
}

(Communicated by Maurice Auslander)

\begin{abstract}
Given a Morita context $(R, S, V, W, \theta, \psi)$, we investigate the relationship between the various homological dimensions of the rings $R$ and $S$. We then apply these results to two particular examples: subidealizers and fixed rings.
\end{abstract}

Let $R$ and $S$ be rings, ${ }_{R} V_{S},{ }_{S} W_{R}$ bimodules and $\theta: V \otimes_{S} W \rightarrow R$ and $\psi: W \otimes_{R} V \rightarrow S$ bimodule homomorphisms. Consider the array

$$
T=\left(\begin{array}{cc}
R & V \\
W & S
\end{array}\right) \text {. }
$$

If $\theta$ and $\psi$ satisfy the associativity conditions required to make $T$ a ring, then we call the collection $(R, S, V, W, \theta, \psi)$ a Morita context and $T$ the ring of the Morita context (see [McCR] for details). Recently, in [C] and [LS], Morita contexts have been used as a tool to relate properties of a ring $R$ and its fixed subring $R^{G}$, where $G$ is a group of automorphisms of $R$. In this paper we also use Morita contexts as a device to obtain information on the homological dimensions of fixed rings and subidealizers.

In the first section, we relate the various homological dimensions of the rings $R$ and $T$ (in a Morita context) under certain assumptions on $V$ or $V$ and $W$ (Lemma 1.2 and Theorem 1.8). If, in addition $\theta$ is onto, it is known that $T$ and $S$ are Morita equivalent (see [LS, Lemma 1.2]). Thus we have a relationship between the homological dimensions of $R$ and $S$.

In $\S 2$, we apply these results to two naturally occurring Morita contexts. In the first example we show that if $S$ is a subidealizer of a generative right ideal $I$ of $R$ (i.e., $S$ is a subring of $R$ containing $I$ as a two-sided ideal and $R I=R$ ), then

$$
\text { w. } \operatorname{gldim}(R) \leq \mathrm{w} \cdot \operatorname{gldim}(S) \leq \max \{\beta+1 \text {, w. } \operatorname{gldim}(R)\},
$$

where $\beta=w . g l d i m(S / I)+w \operatorname{dim}(R / I)_{R}$ (Theorem 2.1), and w. $g l d i m(X)$ denotes the weak global dimension of the ring $X$. Robson and Small [RS] have

Received by the editors December 7, 1989.

1980 Mathematics Subject Classification (1985 Revision). Primary 16A26, 16A60. 
proved the same result for global dimension. As a corollary we show that if $S / I$ is von Neumann regular, then (Corollary 2.2)

$$
\text { w. } \operatorname{gldim}(R) \leq \mathrm{w} \cdot \operatorname{gldim}(S) \leq \mathrm{w} \cdot \operatorname{gldim}(R)+1 .
$$

This result improves on [G, Theorem 2.3].

Our second application involves the fixed ring $R^{G}$ of a ring $R$, where $G$ is a finite group of automorphisms of $R$. Recently, Lorenz [L] has obtained a bound on the global dimension of $R^{G}$ under certain assumptions on $R$ and $G$, including $R$ flat over $R^{G}$. We consider the classical case where the order of $G$ is a unit in $R$; then, if $R$ is flat over $R^{G}$, we show that

$$
\text { r. } \operatorname{gldim}\left(R^{G}\right) \leq \mathrm{r} \cdot \operatorname{gldim}(R),
$$

where r. $\operatorname{gldim}(A)$ denotes the right global dimension of the ring $A$.

Throughout the first section we will assume that we have a fixed Morita context $(R, S, V, W, \theta, \psi)$ with $T$ the ring of the Morita context. To shorten notation we will use $v w$ (resp. $w v$ ) to denote $\theta(v \otimes w)$ (resp. $\psi(w \otimes v)$ ). Moreover, $V W$ (resp. $W V$ ) will be the image of $\theta$ (resp. $\psi$ ). We let $H$ denote the right ideal $\left(\begin{array}{ll}R & V \\ 0 & 0\end{array}\right)$ of $T$. Clearly $H$ is an $R-T$ bimodule, which is projective as a $T$-module. Similarly $K$ will be the left ideal $\left(\begin{array}{ll}R & 0 \\ W & 0\end{array}\right)$ of $T$, which is a $T-R$ bimodule and is also projective as a $T$-module. We note the following useful fact for future reference: $H \otimes_{T} K \approx R$ as $R-R$ bimodules [LS, Proof of Lemma 2.1].

Given a module $M$ over $A$, the projective (resp. weak or flat) dimension of $M$ will be denoted by $\operatorname{pdim}(M)_{A}\left(\operatorname{resp} . \operatorname{wdim}(M)_{A}\right)$. If there is no chance for confusion the subscript will be omitted. Finally, if $M$ is a module and $B$ a set, then $M^{(B)}$ denotes the direct sum of $M$ indexed by $B$.

\section{Dimensions in a Morita context}

In this section we prove our basic results relating the various homological dimensions of the rings $R$ and $T$ in a general Morita context. This will be done in two steps. First we show that the dimension of $R$ is bounded by the dimension of $T$ if $V$ is flat as a left $R$-module. Then we obtain a bound for the dimension of $T$ in terms of $R$ and $S / W V$. The main tools that we will use are the functors $\otimes_{R} H$ from $\operatorname{Mod}-R$ to $\operatorname{Mod}-T$ and ${ }_{-} \otimes_{T} K$ from $\operatorname{Mod}-T$ to $\operatorname{Mod}-R$.

Lemma 1.1. If $M$, as a $T$-module, is the homomorphic image of $H^{(B)}$, for some $B$, then $M$ projective (respectively flat) over $T$ implies that $M \otimes_{T} K$ is projective (resp. flat) over $R$.

Proof. There exists an exact sequence of $T$-modules

$$
0 \rightarrow A \stackrel{g}{\longrightarrow} H^{(B)} \rightarrow M \rightarrow 0 .
$$

If $M$ is projective, this sequence splits and the projective case follows from the fact that $H \otimes_{T} K \approx R$. For the flatness part, since $H$ is projective, we 
can use Villamayor's characterization of flat modules (see, for example, [R, Theorem 3.57]). First we get the exact sequence

$$
0 \rightarrow A \otimes_{T} K \rightarrow R^{(B)} \rightarrow M \otimes_{T} K \rightarrow 0 .
$$

Then let $v=\sum a_{i} \otimes k_{i} \in A \otimes_{T} K$. Since $M$ is flat and $H^{(B)}$ is projective over $T$, there exists $f \in \operatorname{Hom}_{T}\left(H^{(B)}, A\right)$ such that $f\left(g\left(a_{i}\right)\right)=a_{i}$. Let $h=f \otimes 1_{K}$, then $h\left(g \otimes 1_{K}(v)\right)=v$. Therefore $M \otimes_{T} K$ is flat as an $R$-module.

Lemma 1.2. If $V$ is flat as a left $R$-module, then $r \cdot \operatorname{gldim}(R) \leq \mathrm{r} \cdot \operatorname{gldim}(T)$ and w. $\operatorname{gldim}(R) \leq \mathrm{w} \cdot \operatorname{gldim}(T)$.

Proof. We prove the global dimension result (the proof for the weak global dimension is similar). Clearly we can assume that $\mathrm{r}$. $\operatorname{gldim}(T)=m<\infty$. Let $N$ be a right $R$-module. There is an exact sequence of $R$-modules

$$
0 \rightarrow I \rightarrow R^{(A)} \rightarrow N \rightarrow 0
$$

which, since ${ }_{R} V$ is flat, induces the exact sequence of $T$-modules

$$
0 \rightarrow I \otimes_{R} H \rightarrow H^{(A)} \stackrel{\psi}{\longrightarrow} N \otimes_{R} H \rightarrow 0 .
$$

From this we deduce that every $T$-module of the form $X \otimes_{R} H$, where $X$ is an $R$-module, is the homomorphic image of $H^{(A)}$, such that the kernel also has the form $Y \otimes_{R} H$, where $Y$ is an $R$-module. Thus, for any $R$-module $N$, we obtain a long exact sequence of $T$ modules

$$
0 \rightarrow P \rightarrow H^{\left(A_{m-1}\right)} \rightarrow \cdots \rightarrow H^{\left(A_{0}\right)} \rightarrow N \otimes_{R} H \rightarrow 0 .
$$

Since $H$ is a projective $T$-module and since $\mathrm{r} \cdot \operatorname{gldim}(T)=m, P$ must be projective. Also by the above considerations $P$ is a homomorphic image of some $H^{(B)}$. Now applying the exact functor $\otimes_{T} K$ to the above sequence and using the fact that $H \otimes_{T} K \approx R$, we get the sequence of $R$-modules

$$
0 \rightarrow P \otimes_{T} K \rightarrow R^{\left(A_{m-1}\right)} \rightarrow \cdots \rightarrow R^{\left(A_{0}\right)} \rightarrow N \rightarrow 0 .
$$

By Lemma 1.1, $P \otimes_{T} K$ is a projective $R$-module. Therefore $\operatorname{pdim}(N) \leq m$ and hence the lemma is proved.

We will now work toward getting a bound on the dimensions of the ring $T$ in terms of the dimension of the rings $R$ and $S / W V$ and the dimension of the $S$-module $S / W V$.

Lemma 1.3. Let $M$ be a T-module which is killed by the ideal $I$. Then

$$
\operatorname{pdim}(M) \leq \mathrm{r} \cdot \operatorname{gldim}(T / I)+\operatorname{pdim}(T / I)_{T}
$$

and

$$
w \operatorname{dim}(M) \leq \mathrm{w} \cdot \operatorname{gldim}(T / I)+\operatorname{wdim}(T / I)_{T} .
$$

Proof. The global dimension part is well known (see, for example, [R, Theorem 9.32]). The weak dimension part has almost the same proof except at the start of the induction (on $\left.\operatorname{wdim}(M)_{T / I}\right)$. To deal with that case, first recall a 
result of Lazard which says that a flat module is a direct limit of projective modules (see, for example, [F, Proposition 11.32]). The result then follows from the fact that Tor commutes with direct limits.

The ideal $\left(\begin{array}{cc}R & V \\ W & W V\end{array}\right)$ of $T$ will be denoted by $J$. It is not difficult to see that the rings $T / J$ and $S / W V$ are isomorphic.

Lemma 1.4. Assume that the left $S$-module $W$ is flat and that $(W V) W=W$, then $\operatorname{pdim}(T / J)_{T} \leq \operatorname{pdim}(S / W V)_{S}$ and $\operatorname{wdim}(T / J)_{T} \leq w \operatorname{dim}(S / W V)_{S}$.

Proof. We prove the projective dimension result (the proof for the weak dimension is similar). Again we can assume that $\operatorname{pdim}(S / W V)_{S}=n<\infty$. Take an $S$-projective resolution of $S / W V$

$$
0 \rightarrow P_{n} \rightarrow \cdots \rightarrow P_{0} \rightarrow S / W V \rightarrow 0 .
$$

Denote the right ideal $\left(\begin{array}{cc}0 & 0 \\ W & S\end{array}\right)$ of $T$ by $H^{\prime}$. By our assumption on $W, H^{\prime}$ is flat as a left $S$-module. So we get the exact sequence of $T$-modules

$$
0 \rightarrow P_{n} \otimes_{S} H^{\prime} \rightarrow \cdots \rightarrow P_{0} \otimes_{S} H^{\prime} \rightarrow(S / W V) \otimes_{S} H^{\prime} \rightarrow 0 .
$$

Since $H^{\prime}$ is a projective $T$-module, so is $P_{i} \otimes_{S} H^{\prime}$ and hence

$$
\operatorname{pdim}\left((S / W V) \otimes_{S} H^{\prime}\right)_{T} \leq n
$$

(for the weak dimension, observe that $P_{i} \otimes_{S} H^{\prime}$ is a flat $T$-module whenever $P_{i}$ is a flat $S$-module).

From the short exact sequence

$$
0 \rightarrow W V \rightarrow S \rightarrow S / W V \rightarrow 0
$$

we obtain the exact sequence

$$
0 \rightarrow W V \otimes_{S} H^{\prime} \rightarrow H^{\prime} \rightarrow(S / W V) \otimes_{S} H^{\prime} \rightarrow 0 .
$$

It follows that $(S / W V) \otimes_{S} H^{\prime}$ is isomorphic to $H^{\prime} / W V H^{\prime}$ as $T$-modules. However, $W V H^{\prime}=\left(\begin{array}{cc}0 & 0 \\ W & W V\end{array}\right)$, since $(W V) W=W$. Thus one can see that $T / J$ is isomorphic to $H^{\prime} / W V H^{\prime}$ and this proves the result.

Remark 1.5. Clearly it follows from Lemmas 1.3 and 1.4 that if $W$ is a flat $S$-module and if $(W V) W=W$, then for any right $T$-module $M$ such that $M J=0$ we can conclude that

$$
\operatorname{pdim}(M)_{T} \leq \mathrm{r} \cdot \operatorname{gldim}(S / W V)+\operatorname{pdim}(S / W V)_{S},
$$

and

$$
w \operatorname{dim}(M)_{T} \leq \mathrm{w} \cdot \operatorname{gldim}(S / W V)+\mathrm{wdim}(S / W V)_{S} .
$$

From now on we will denote the right side of the first inequality by $\alpha$, and the right side of the second by $\alpha^{\prime}$.

There is a natural setting for when the conditions of Lemma 1.4 are satisfied. Namely, if $V W=R$, then $W$ is a finitely generated projective left $S$ module, since it has a projective basis (let $\sum v_{i} w_{i}=1$ and then let $f_{i}: W \rightarrow S$ be defined by $f(w)=w v_{i}$, then the $f_{i}$ 's and the $w_{i}$ 's form a projective basis). Similarly, $V$ is a finitely generated projective right $S$ module. Furthermore $(W V) W=W(V W)=W$. This setting will be used in $\S 2$. 
Lemma 1.6. Let $V$ be flat as a left $R$-module. Then for any right $R$-module $N, \operatorname{pdim}\left(N \otimes_{R} H\right)_{T} \leq \operatorname{pdim}(N)_{R}$ and $\operatorname{wdim}\left(N \otimes_{R} H\right)_{T} \leq \operatorname{wdim}(N)_{R}$.

Proof. As in Lemma 1.4 we only have to prove the projective dimension case. Again we can assume that $\operatorname{pdim}(N)_{R}=n<\infty$. Thus we have a projective resolution of the $R$-module $N$

$$
0 \rightarrow P_{n} \rightarrow \cdots \rightarrow P_{0} \rightarrow N \rightarrow 0
$$

Since $H$ is flat as a left $R$-module, we have

$$
0 \rightarrow P_{n} \otimes_{R} H \rightarrow \cdots \rightarrow P_{0} \otimes_{R} H \rightarrow N \otimes_{R} H \rightarrow 0 .
$$

As in the proof of Lemma 1.4, it is clear that $P_{i} \otimes_{R} H$ is projective as a $T$ module and hence $\operatorname{pdim}\left(N \otimes_{R} H\right)_{T} \leq n$.

Before we get to the main result of the section, we need some facts relating the projective and weak dimensions of modules in an exact sequence. For a module $M$, both dimensions will be denoted $\operatorname{dim}(M)$. Consider the short exact sequence of modules

$$
0 \rightarrow B \rightarrow A \rightarrow C \rightarrow 0
$$

Then

(1) If $\operatorname{dim}(A)>\operatorname{dim}(B)$, then $\operatorname{dim}(C)=\operatorname{dim}(A)$

(2) If $\operatorname{dim}(A) \leq \operatorname{dim}(B)$, then $\operatorname{dim}(C) \leq \operatorname{dim}(B)+1$.

For the projective dimension results, see, for example, [K, Part III, Theorem 2]. The weak dimension results can be proved in the same fashion using Tor instead of Ext. In particular one can conclude that

$$
\operatorname{dim}(C) \leq \max \{\operatorname{dim}(B)+1, \operatorname{dim}(A)\} .
$$

Lemma 1.7. Let $0 \rightarrow M \rightarrow N \stackrel{f}{\longrightarrow} P \rightarrow Q \rightarrow 0$ be an exact sequence of right modules. Then $\operatorname{dim}(P) \leq \max \{\operatorname{dim}(M)+1, \operatorname{dim}(Q), \operatorname{dim}(N)\}$.

Proof. Let $U$ be the cokernel of $f$. By the above,

$$
\operatorname{dim}(U) \leq \max \{\operatorname{dim}(M)+1, \operatorname{dim}(N)\} .
$$

The bound on $\operatorname{dim}(P)$ now follows from a standard argument using Tor or Ext .

We are now able to prove the main result of this section, which gives a bound on the dimensions of $T$.

Theorem 1.8. Let $V$ and $W$ be flat as left $R$-and $S$-modules, respectively, and let $(W V) W=W$. Then

$$
\max \{\mathrm{r} \cdot \operatorname{gldim} R, \mathrm{r} \cdot \operatorname{gldim} S\} \leq \mathrm{r} \cdot \operatorname{gldim}(T) \leq \max \{\alpha+1, \mathrm{r} \cdot \operatorname{gldim}(R)\}
$$

and

$\max \{$ w. $\operatorname{gldim} R$, w. $\operatorname{gldim} S\} \leq$ w. $\operatorname{gldim}(T) \leq \max \left\{\alpha^{\prime}+1\right.$, w. $\left.\operatorname{gldim}(R)\right\}$, where $\alpha$ and $\alpha^{\prime}$ are given in Remark 1.5. 
Proof. As usual we only prove the global dimension case. The left-hand inequality is Lemma 1.2. For the other inequality, let $M$ be any right $T$-module. From the short exact sequence

$$
0 \rightarrow J \rightarrow T \rightarrow T / J \rightarrow 0
$$

we get the exact sequence of right $T$-modules

$$
0 \rightarrow \operatorname{Tor}_{T}^{1}(M, T / J) \rightarrow M \otimes_{T} J \rightarrow M \rightarrow M \otimes_{T}(T / J) \rightarrow 0 .
$$

First we need bounds on the dimension of $M \otimes_{T} J$. Consider the surjection $f$ : $K \otimes_{R} H \rightarrow J$ given by multiplication $k \otimes h \rightarrow k h$.

Claim. (Kerf) $J=0$.

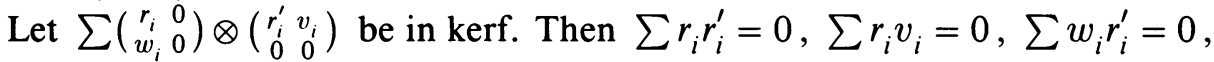
and $\sum w_{i} v_{i}=0$. Let $\left(\begin{array}{cc}r & v \\ w & x y\end{array}\right) \in J$, where $x \in W$ and $y \in V$. Then

$$
\begin{aligned}
\left(\sum\left(\begin{array}{cc}
r_{i} & 0 \\
w_{i} & 0
\end{array}\right) \otimes\left(\begin{array}{cc}
r_{i}^{\prime} & v_{i} \\
0 & 0
\end{array}\right)\right)\left(\begin{array}{cc}
r & v \\
w & x y
\end{array}\right) \\
=\sum\left(\begin{array}{cc}
r_{i} & 0 \\
w_{i} & 0
\end{array}\right) \otimes\left(\begin{array}{cc}
r_{i}^{\prime} r+v_{i} w & r_{i}^{\prime} v+v_{i} x y \\
0 & 0
\end{array}\right) \\
=\sum\left(\begin{array}{cc}
r_{i} & 0 \\
w_{i} & 0
\end{array}\right) \otimes\left(\begin{array}{cc}
r_{i}^{\prime} r & r_{i}^{\prime} v \\
0 & 0
\end{array}\right)+\sum\left(\begin{array}{cc}
r_{i} & 0 \\
w_{i} & 0
\end{array}\right) \otimes\left(\begin{array}{cc}
v_{i} w & 0 \\
0 & 0
\end{array}\right) \\
\quad+\sum\left(\begin{array}{ll}
r_{i} & 0 \\
w_{i} & 0
\end{array}\right) \otimes\left(\begin{array}{cc}
0 & \left(v_{i} x\right) y \\
0 & 0
\end{array}\right) .
\end{aligned}
$$

Since the tensor product is over $R$, each of the three sums is zero. This proves the claim.

Clearly the kernel $A$ of the induced surjection

$$
M \otimes_{T} K \otimes_{R} H \rightarrow M \otimes_{T} J
$$

is also killed by $J$. By Remark $1.5 \operatorname{pdim}(A) \leq \alpha$ and by Lemma 1.6

$$
\operatorname{pdim}\left(M \otimes_{T} K \otimes_{R} H\right) \leq \mathrm{r} \cdot \operatorname{gldim}(R) .
$$

So from the discussion prior to Lemma 1.7,

$$
\operatorname{pdim}\left(M \otimes_{T} J\right) \leq \max \{\alpha+1, \text { r. } \operatorname{gldim}(R)\} .
$$

Now we return to sequence $(*)$. The modules $\operatorname{Tor}_{T}^{1}(M, T / J)$ and $M \otimes_{T}(T / J)$ are killed by $J$, and hence, by Remark 1.5 , both of these modules have projective dimension less than or equal to $\alpha$. Therefore, by Lemma 1.7, $\operatorname{pdim}\left(M_{T}\right) \leq \max \{\alpha+1, \mathrm{r}$. $\operatorname{gldim}(R)\}$. Hence the result is proved.

As noted after Remark 1.5, if $V W=R$ and if ${ }_{R} V$ is flat, then the hypotheses of Theorem 1.8 are satisfied. Also in this case $T$ and $S$ are Morita equivalent ([LS, Lemma 1.2]). Thus we have the following results:

$$
\text { r. } \operatorname{gldim}(S) \leq \max \{\alpha+1, \text { r. } \operatorname{gldim}(R)\}
$$


and

$$
\text { w. } \operatorname{gldim}(S) \leq \max \left\{\alpha^{\prime}+1, \text { w. } \operatorname{gldim}(R)\right\} .
$$

If $S$ is any ring and $e \in S$ is an idempotent, then (eSe, $S, e S, S e, \theta, \psi)$, where $\theta$ and $\psi$ are multiplication, is a Morita context. So when $e S$ is flat as a left $e S e$-module, the above result is an improvement on [KK, Proposition 2.6].

\section{APPLICATIONS TO IDEALIZERS AND FIXED RINGS}

There are two natural examples of Morita contexts to which we want to apply the results of the previous section. For the first, let $I$ be a right ideal of the ring $R$ and let $S$ be a subring of $R$ which contains $I$ as a two-sided ideal. Then $S$ is called a subidealizer of $I$ in $R$. In the notation of the last section, if we let $V=R$ and $W=I$, then

$$
T=\left(\begin{array}{ll}
R & R \\
I & S
\end{array}\right) \text { is a Morita context. }
$$

We are able to use the results of the last section to relate $w$. $\operatorname{gldim}(R)$ and w. gldim $(S)$. There have been a number of papers relating the global dimension of $R$ and $S$. First Goodearl [G] had some results under the assumption that $S / I$ is semisimple. Teply [T] and Hansen [H] obtained a generalization of this result which eliminated the semisimple assumption. More recently Robson and Small [RS, Theorems 2 and 5] have further improved on this in a number of directions. In our next result we use Theorem 1.8 to give relations between the weak global dimensions of $R$ and $S$, which are the same as the ones obtained for the right global dimension in [RS, Theorem 5] (note that we can also get their relations from Theorem 1.8). Before we give the theorem let us recall that a right ideal $I$ of $R$ is said to be generative if $R I=R$. In this case, using the notation of the last section, we have $V W=R$ and $W V=I$.

Theorem 2.1. Let $I$ be a generative right ideal of $R$. If $S$ is a subidealizer of $I$ in $R$, then

$$
\begin{aligned}
\mathrm{w} \cdot \operatorname{gldim}(R) & \leq \mathrm{w} \cdot \operatorname{gldim}(S) \\
& \leq \max \left\{\mathrm{w} \cdot \operatorname{gldim}(S / I)+\mathrm{wdim}(R / I)_{R}+1, \mathrm{w} \cdot \operatorname{gldim}(R)\right\} .
\end{aligned}
$$

Proof. Since $I$ is generative, as we have noted before, $T$ and $S$ are Morita equivalent. So by $\operatorname{Lemma} 1.2$, w. $\operatorname{gldim}(R) \leq \mathrm{w} \cdot \operatorname{gldim}(S)$. Clearly $V=R$ is flat as a left $R$-module, and since $V W=R$, the hypotheses of Theorem 1.8 are satisfied. By [G, Proposition 1.2], $w \operatorname{dim}(I)_{R} \leq w \operatorname{dim}(I)_{S}$. Furthermore, since $V W=R, V=R$ is projective as an $S$-module, hence any flat $R$-module is a flat $S$-module by Lazard's Theorem. This gives us the reverse inequality. So $w \operatorname{dim}(S / I)_{S}=\operatorname{wdim}(R / I)_{R}$ and thus the result follows from Theorem 1.8.

Finally, as a corollary we obtain an improvement of a result of Goodearl in [G].

Corollary 2.2. Under the previous assumptions, if in addition $S / I$ is a von Neumann regular ring, then $\mathrm{w} \cdot \operatorname{gldim}(R) \leq \mathrm{w} \cdot \operatorname{gldim}(S) \leq \mathrm{w} \cdot \operatorname{gldim}(R)+1$.

Proof. The result follows immediately from the fact that $w \cdot \operatorname{gldim}(S / I)=0$. 
Our second application arises in the Galois theory of noncommutative rings. Let $G$ be a finite group of automorphisms acting on the ring $R$. The fixed ring $R^{G}$ is defined to be the subring $\left\{x \in R \mid x^{g}=x\right.$ for all $\left.g \in G\right\}$. If $|G|^{-1} \in R$, then, as noted in [L, 2.2], it is well known that

$$
\text { r. } \operatorname{gldim}\left(R^{G}\right) \leq \mathrm{r} \cdot \operatorname{gldim}(R)+\operatorname{pdim}(R)_{R^{G}} .
$$

Lorenz replaced the assumption that $|G|^{-1} \in R$ by other, more general, assumptions on $R$ and $G$. He then obtains certain bounds on r. $\operatorname{gldim}\left(R^{G}\right)$ when $R$ is flat as a left $R^{G}$-module. We stay in the classical case where $|G|$ is a unit in $R$; in addition we use Lorenz's assumption that $R$ is flat as a left $R^{G}$-module. Then we improve on $(*)$ by showing that

$$
\text { r. } \operatorname{gldim}\left(R^{G}\right) \leq \text { r. } \operatorname{gldim}(R) .
$$

Let $R$ and $G$ be as before. The skew group ring $R * G$ is the set of all formal sums $\sum_{g \in G} r_{g} g, r_{g} \in R$. Addition is componentwise and multiplication is defined distributively by the formula

$$
r g \cdot s h=r s^{g^{-1}} g h,
$$

for $r, s \in R$ and $g, h \in G$. Clearly $R$ is a left and right module over $R^{G}$. $R$ can also be viewed as a left or right $R * G$-module as follows: for any $s=$ $\sum r_{g} g \in R * G$ and $r \in R$, define $s \cdot r=\sum r_{g} r^{g^{-1}}$ and $r \cdot s=\sum\left(r r_{g}\right)^{g}$. Then $T=\left(\begin{array}{cc}R^{G} & R \\ R * G\end{array}\right)$ is a Morita context. See [C] for details. We note that the map $\psi: R \otimes_{R^{G}} R \rightarrow R * G$ is defined by $\psi(x \otimes y)=\sum_{g \in G} x y^{g^{-1}} g$. So it is not difficult to see that the image of $\psi$ is the two-sided ideal generated by the element $\sum_{g \in G} g$. We will denote this ideal by $L$. The map $\theta: R \otimes_{R * G} R \rightarrow R^{G}$ is given by $\theta(x \otimes y)=\sum_{g \in G}(x y)^{g}$ and if $|G|^{-1} \in R$, then $\theta$ is easily seen to be onto, i.e., in the notation of $\S 1, V W=R^{G}$.

Theorem 2.3. If $|G|^{-1} \in R$ and if $R$ is flat as a left $R^{G}$-module, then

$$
\text { r. } \operatorname{gldim}\left(R^{G}\right) \leq \mathrm{r} \cdot \operatorname{gldim}(R) \leq \max \left\{\alpha+1, \mathrm{r} \cdot \operatorname{gldim}\left(R^{G}\right)\right\},
$$

where $\alpha=\mathrm{r} \cdot \operatorname{gldim}(R * G / L)+\operatorname{pdim}(R * G / L)_{R * G}$.

Proof. Since $V W=R^{G}, T$ and $R * G$ are Morita equivalent. Also, by [McCR, Theorem 7.5.6], $R$ is projective as a left $R * G$-module and $\mathrm{r} \cdot \operatorname{gldim}(R * G)=$ r. $\operatorname{gldim}(R)$. So, by Lemma 1.2 and Theorem 1.8, we are done.

Note that the above result does not include a statement about weak global dimension, since the assumptions easily imply, from other considerations, that w. $\operatorname{gldim}\left(R^{G}\right) \leq \mathrm{w} \cdot \operatorname{gldim}(R)$ (namely, if $|G|$ is a unit in $R$, then one can show, as in the global dimension case, that $\left.\mathrm{w} \cdot \operatorname{gldim}\left(R^{G}\right) \leq \mathrm{w} \cdot \operatorname{gldim}(R)+\mathrm{wdim}(R)_{R^{G}}\right)$.

In light of the hypothesis in Theorem 2.3 it is natural to ask, when is $R$ flat over the fixed ring $R^{G}$ ? There does not seem to be a great deal of work on 
this question though there are some situations in which this happens naturally. There are a number of cases where $R$ is projective over $R^{G}$ (see, for example, [L]). Though, in view of Theorem 2.3 and [L, 2.2], it would be more interesting to know when $R$ is merely flat over $R^{G}$.

If $R$ is regular, then it is well known that $R^{G}$ is regular if the order of $G$ is a unit in $R$. So, in this case, both parts of the hypothesis of Theorem 2.3 are satisfied. Furthermore, Jøndrup [J, Examples 4 and 5] has given examples where $R$ is regular and $|G|^{-1} \in R$, yet $R$ is not projective over $R^{G}$. For another example recall that a commutative domain is called a Prufer domain if every finitely generated ideal is invertible (equivalently, projective). Now assume that $R$ is such a domain with quotient field $Q$. Let $I$ be a finitely generated right ideal of $R^{G}$. Since $I R$ is a finitely generated ideal of $R$, there exists $b_{i} \in I R$ and $q_{i} \in(I R)^{*}=\{q \in Q \mid I R q \subseteq R\}, i=1, \ldots, n$, such that $\sum_{1}^{n} b_{i} q_{i}=1$. Since $b_{i} \in I R$ and since $(I R)^{*}$ is an $R$-submodule of $Q$, we can assume that $b_{i} \in I$ (though $q_{i}$ is only in $\left.(I R)^{*}\right)$. We then get

$$
\sum_{g \in G}\left(\sum_{1}^{n} b_{i} q_{i}^{g}\right)=|G| 1,
$$

which, by rearranging terms, becomes

$$
\sum_{1}^{n} b_{i}\left(\sum_{g \in G} q_{i}^{g}\right)=|G| 1 .
$$

For a fixed $i$, the element $\sum_{g \in G} q_{i}^{g}$ is in $Q^{G}$, which, if $|G|^{-1} \in R$, is well known to be the quotient field of $R^{G}$. Moreover, this element is in $I^{*}$, so if $|G|^{-1} \in R$, it follows that $I$ is invertible, i.e., $R^{G}$ is also a Prufer domain. Clearly $R$ is torsion free as a module over $R^{G}$ and, since torsion-free modules over a Prufer domain are necessarily flat, we have another class of examples where $R$ is flat over $R^{G}$.

Finally we give a simple example to show that the first inequality in Theorem 2.3 can be strict. Let $K$ be a field of characteristic not two and let $g$ be the automorphism of $K[x]$ which sends $f(x)$ to $f(-x)$. Clearly the ideal $I$ generated by $x^{2}$ is $g$-invariant. Hence $g$ defines an automorphism of $R=K[x] / I$. Let $G=\langle g\rangle$, then $R^{G}=K$. Clearly $R^{G}$ has global dimension 0 and $R$ is flat over $R^{G}$, yet $R$ has infinite global dimension.

\section{ACKNOWLEDGMENT}

The authors are deeply indebted to Professors James Kuzmanovich and Mark Teply for their helpful comments. 


\section{REFERENCES}

[C] M. Cohen, A Morita context related to finite automorphism groups of rings, Pacific J. Math. 98 (1982), 37-54.

[F] C. Faith, Algebra: rings, modules and category I, Springer-Verlag, New York, 1973.

[G] K. R. Goodearl, Subrings of idealizer rings, J. Algebra 33 (3) (1975), 405-429.

[H] F. Hansen, Ringerweiterung und lokalisation, Comm. Algebra 7 (1979), 689-751.

[J] S. Jøndrup, When is the ring a projective module over the fixed point ring?, Comm. Algebra 16 (1988), 1971-1992.

[K] I. Kaplansky, Fields and rings, Chicago Lectures in Mathematics, Chicago, 1972.

[KK] E. Kirkman and J. Kuzmanovich, Global dimensions of a class of tiled orders, J. Algebra (to appear).

[L] M. Lorenz, On the global dimension of fixed rings, preprint.

[LS] P. Loustaunau and J. Shapiro, Localization in a Morita context with application to fixed rings, preprint.

[McCR] J. C. McConnell and J. C. Robson, Noncommutative noetherian rings, Wiley Series in Pure and Appl. Math., New York, 1987.

[R] J. Rotman, An introduction to homological algebra, Academic Press, Orlando, 1979.

[RS] J. C. Robson and L. W. Small, Another change of rings theorem, Bull. London Math. Soc. 20 (1988), 297-301.

[T] M. L. Teply, Subidealizers, Module Theory: Papers and Problems, Proceedings (Seattle 1977), Lecture Notes in Math., vol. 700, Springer-Verlag, Berlin, 1979, pp. 232-234.

Department of Mathematics, George Mason University, Fairfax, Virginia 22030 